\title{
Sliding Contact Using Electroconductive Liquid and Its Application to Low-physical-restriction Micropotentiometer
}

\author{
Satoshi Konishi* \\ Department of Mechanical Engineering, College of Science and Engineering, \\ Ritsumeikan University, 1-1-1 Noji-higashi, Kusatsu, Shiga 525-8577, Japan \\ (Received December 26, 2018; accepted March 18, 2019)
}

Keywords: electroconductive liquid, sliding contact, potentiometer, ionic liquid, sliding friction

In this paper, a sliding electrical contact with an electroconductive liquid is presented. We have proposed a sliding contact using a combination of an electroconductive liquid and a dipped probe electrode. The proposed connection can overcome the drawback of a conventional electrical connection, such as the suspension spring for microactuators, and enables the connection distance to be extended without increasing restriction. As a typical application of the sliding contact, a low-physical-restriction micropotentiometer is designed. Conventional potentiometers have physical restrictions or sliding friction caused by a sliding contact of a variable electrical resistance. It is not practical to apply conventional potentiometers having a high sliding friction to measure the motion of microactuators. The proposed micropotentiometer can significantly reduce the physical restrictions at the contact point of variable resistances. Moreover, the proposed micropotentiometer can detect displacements of 2 $\mu \mathrm{m}$ with good linearity. The measured restriction force is a few $\mathrm{mN}$ when the probe is moved in an electroconductive liquid at $5 \mu \mathrm{m} / \mathrm{s}$, whereas the value for a commercial potentiometer is more than $100 \mathrm{mN}$.

\section{Introduction}

Various microsensors and microactuators have been designed and developed in the field of MEMS. In the practical use of microdevices, the implementation technology of a core device and peripheral components are as important as the device performance itself. The wiring technology is one of the key technologies in implementing the components. Microsensors transmit detected signals to processing circuits through the transmission wire and microactuators receive driving energy through the supply wire. Inertia microsensors and microactuators have movable parts where suspension spring structures are often used for wiring. ${ }^{(1)}$ However, the suspension spring has a limitation on its extension. The tension, which depends on the extension of the spring, causes a physical restriction of the motion of microsensors and microactuators. In general, outputs of microactuators, such as displacements and forces, are small. Typical displacements and forces generated by electrostatic comb drive actuators are of

*Corresponding author: e-mail: konishi@se.ritsumei.ac.jp

https://doi.org/10.18494/SAM.2019.2246 
the orders of $\mu \mathrm{m}$ and $\mu \mathrm{N}$, respectively. The suspensions for movable electrodes of electrostatic comb drive actuators are also used to supply driving signals. ${ }^{(2,3)}$ The physical restriction imposed by suspension springs interferes with the motion of microactuators. Moreover, microactuators are controlled by the feedback of their motion. Microsensors are required to detect the motion of microactuators while having a minimal effect on that motion. Several methods have been employed to overcome the problem of physical restriction. For example, the use of optical signal processing (e.g., using an optical encoder) is an effective solution to removing the structural interconnection. ${ }^{(4,5)}$ A position-sensitive device is a commercialized position sensor with a photodiode. However, optical signal processing is at a disadvantage because it requires complicated optical instruments and additional signal processors. ${ }^{(6)}$ It is still important to develop wiring and sensing technology with a low physical restriction.

In this paper, a sliding electrical contact using an electroconductive liquid is presented (Fig. 1). We have proposed a sliding contact using a combination of an ionic liquid and a dipped probe electrode. ${ }^{(7)}$ Recently, some research studies have been reported on the application of an ionic liquid in a MEMS field. Liu et al. reported the application of an ionic liquid for both mechanical bearings and electric connection. ${ }^{(8)}$ Noda et al. reported a capacitive tactile sensor using an ionic liquid. ${ }^{(9)}$ The proposed connection can overcome the drawback of conventional electrical connections, such as a suspension spring for movable microdevices, and enables the extension of the connection distance without increasing restriction. This will allow a wide range of both the sensing of microsensors and the motion of microactuators. In this paper, the micropotentiometer is reported as a typical application of the sliding contact with a low physical restriction. ${ }^{(10)}$ Figure 1 shows our proposed potentiometer using an electroconductive liquid compared with a conventional potentiometer using a solid sliding contact. Potentiometers with variable resistors have been widely used to measure the position of objects. Typically, they

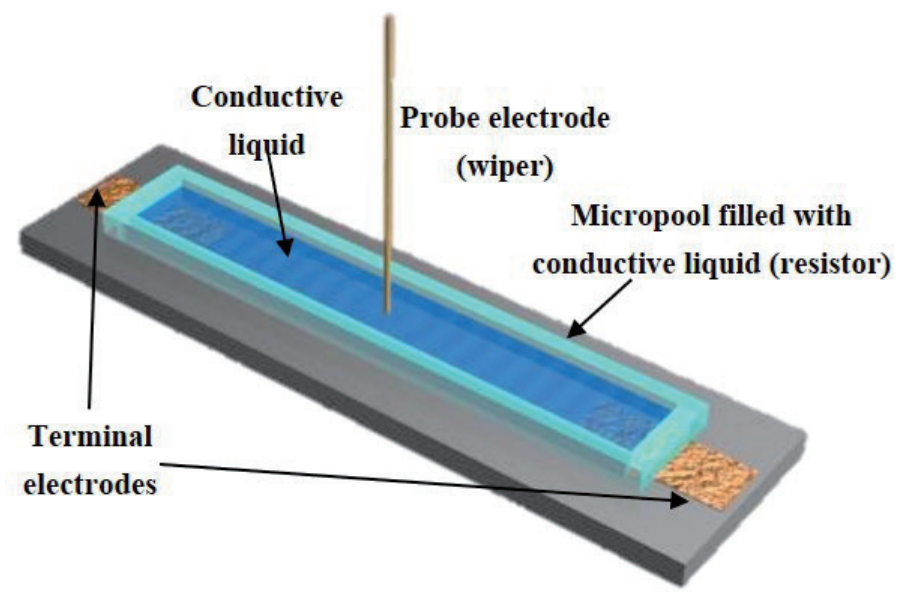

Fig. 1. (Color online) Proposed sliding electrical contact with a probe electrode in a micropool of conductive liquid. 
are composed of an electrical resistance and a wiper for a sliding contact. The resistance has a terminal electrode at each edge. The electrical resistance between the wiper and one of the terminal electrodes changes when the wiper moves along the resistance. However, conventional potentiometers have sliding friction generated by the sliding contact of a variable resistor. When this type of potentiometer is integrated into microactuators, the sliding friction markedly affects the motion of microactuators. The proposed micropotentiometer employs a sliding electrical contact using an electroconductive liquid to reduce the physical restriction at the contact point. The primary restriction of the proposed potentiometer is not sliding friction but the fluid resistance of the conductive liquid in the micropool.

A sliding electrical contact comprising an electroconductive liquid is reported. The design, fabrication, and characterization of a low-physical-restriction micropotentiometer based on the proposed sliding contact are described.

\section{Sliding Electrical Contact with Electroconductive Liquid for Potentiometer}

\subsection{Low-physical-restriction sliding contact with electroconductive liquid}

A sliding electrical contact with an electroconductive liquid has been developed in order to reduce sliding friction at the contact point. The proposed sliding contact employs a probe electrode dipped in a micropool filled with an electroconductive liquid, as shown in Fig. 1. The sliding contact has no physical architecture for the electrical connection because there are no physical restrictions between the probe and the conductive liquid, except for the fluid resistance against the moving probe. Although a guide groove is designed at the bottom of the micropool to avoid the physical contact against the bottom, the probe electrode is thin enough to move along the groove without the contact against the side walls. Consequently, the sliding contact can significantly reduce the physical restrictions imposed by the wiring on a movable electrode. A nonvolatile, ionic liquid, 1-ethyl-3-methylimidazolium trifluoroacetate (MERCK Co., degradation temperature: $130{ }^{\circ} \mathrm{C}$, viscosity: $29.42 \mathrm{cP}$, conductivity: $9.6 \mathrm{mS} / \mathrm{cm}$ ), is employed as the conductive liquid because of its high stability.

\subsection{Micropotentiometer having low physical restriction}

As a typical application of the proposed sliding contact, a low-physical-restriction micropotentiometer is designed. The structure of the proposed sliding contact can act as a potentiometer as well (Fig. 1). The relative position of the probe along the micropool resistor with two terminal electrodes can be determined by measuring the resistance between the probe and one of the terminal electrodes. To the contrary, a conventional potentiometer consists of a solid sliding contact between a wiper electrode and a resistor made of solid materials such as conductive plastic. Conventional potentiometers have a physical restriction or sliding friction caused by a solid sliding contact of a variable resistor. On the other hand, in Fig. 1, the probe electrode and the micropool filled with an electroconductive liquid work as a wiper and a resistor, respectively. The variable resistor is composed of a longitudinal micropool filled with 
a conductive liquid and has a terminal electrode at each edge. The probe electrode as the wiper is dipped into and moves in the micropool. The position of the probe electrode in the micropool is indicated by the electrical resistance between the probe electrode and one of the terminal electrodes. The primary restriction of the proposed potentiometer is fluid resistance due to the conductive liquid in the micropool. The proposed micropotentiometer enables reduced physical restrictions at the contact point of the variable resistor.

\section{Fabrication}

\subsection{Fabrication process}

A low-physical-restriction micropotentiometer with the proposed sliding contact is composed of a probe electrode dipped in a micropool filled with an electroconductive liquid. Figure 2 shows the fabrication process of the micropotentiometer completed by integrating a micropool [Fig. 2(a)] and a probe electrode [Fig. 2(b)] fabricated separately.

The fabrication process of the micropool is explained using Fig. 2(a). A 1550- $\mu \mathrm{m}$-wide and $150-\mu \mathrm{m}$-deep guide groove was etched by deep reactive-ion etching (DRIE) onto the top surface of a Si strip. The Si strip was thermally oxidized and insulated. Two Au terminal electrodes were deposited and patterned on the insulted Si strip. Then, a micropool surrounded by a SU-8 wall was fabricated on the insulated Si strip where the terminal electrodes were aligned at the two edges of the longitudinal micropool. An ionic liquid, 1-ethyl-3-methylimidazolium trifluoroacetate, was introduced into the micropool as the conductive, nonvolatile liquid. Ionic liquids are generally suitable as solvents for electrochemical applications because of their incombustibility and low vapor pressure. The micropool filled with the ionic liquid acts as an electrical resistor. Next, a probe electrode was prepared by a combination of wire bonding and laser machining as explained in Fig. 2(b). ${ }^{(1,12)}$ Both wire bonding and laser machining are widely used as production technologies in industry. An excimer laser was used to cut a Au wire of $20 \mu \mathrm{m}$ diameter. Bonded Au wires were converted into probes by cutting their bridges [Fig. 2(b)]. Finally, the fabricated probe electrode was aligned and implemented on the micropool to complete the fabrication of the micropotentiometer [Fig. 2(c)].

\subsection{Fabrication results}

Figure 3 shows the fabrication results of the microlinear potentiometer. The micropools of the potentiometers could be batch-fabricated as shown in Fig. 3(a). Figure 3(b) shows an enlarged image of a Au probe electrode $(\Phi 20 \mu \mathrm{m})$ dipped in a micropool. A $500 \times 2500 \times 70$ $\mu \mathrm{m}^{3}$ micropool was fabricated on a $1.5 \times 3.75 \mathrm{~mm}^{2}$ Si strip. A $1550-\mu \mathrm{m}$-wide and $150-\mu \mathrm{m}-$ deep guide groove was fabricated in the Si strip. The guide groove was designed at the bottom of the micropool in order to guarantee the linear motion of the probe. The probe electrode moves along the guide groove. A terminal electrode with wide electrode pads was fabricated at each edge of the micropool in Fig. 3(a). The ionic liquid $(0.1 \mu \mathrm{l})$ was then injected into the micropool using a microsyringe. As shown in Fig. 3(b), the probe electrode was aligned with the micropool and was dipped into the micropool. 


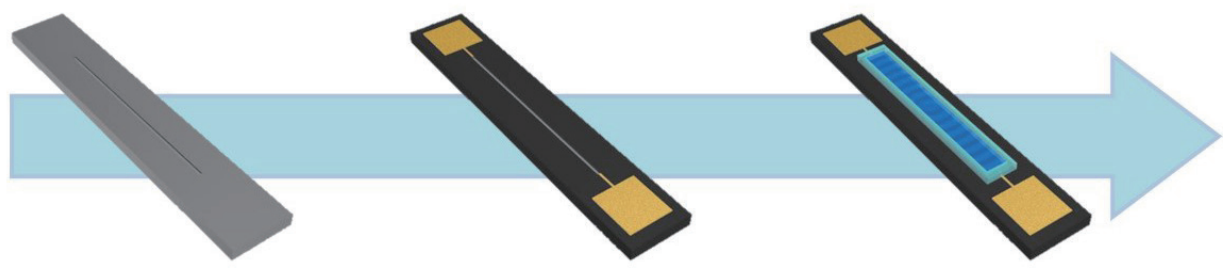

(a)

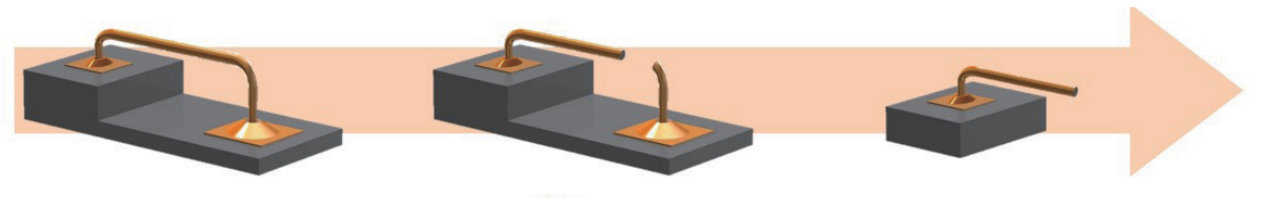

(b)

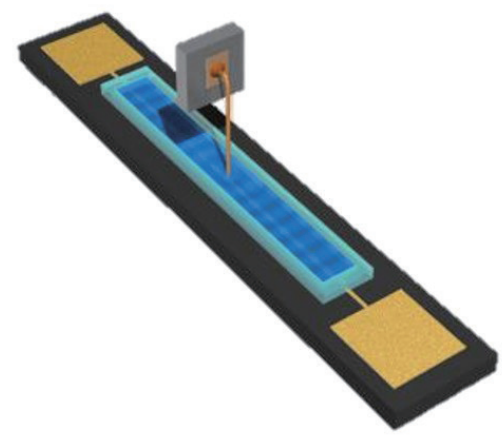

(c)

Fig. 2. (Color online) Fabrication process of individual parts. (a) Fabrication process of micropool. (b) Fabrication process of probe electrode (wiper). (c) Final assembly of micropotentiometer.

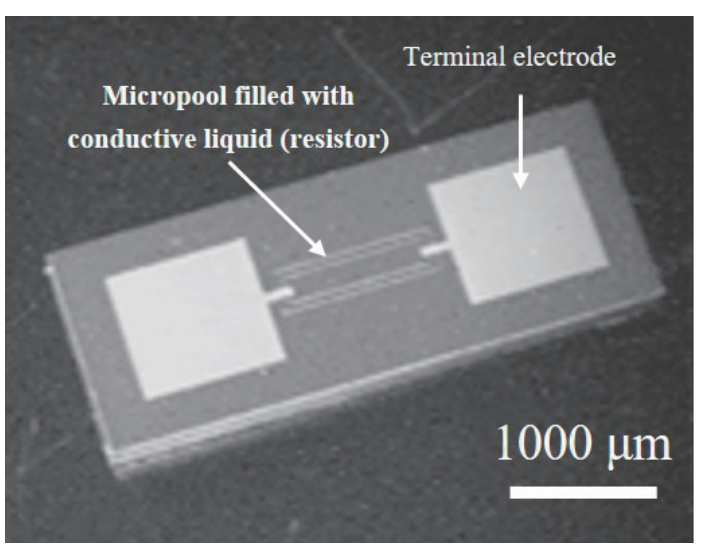

(a)

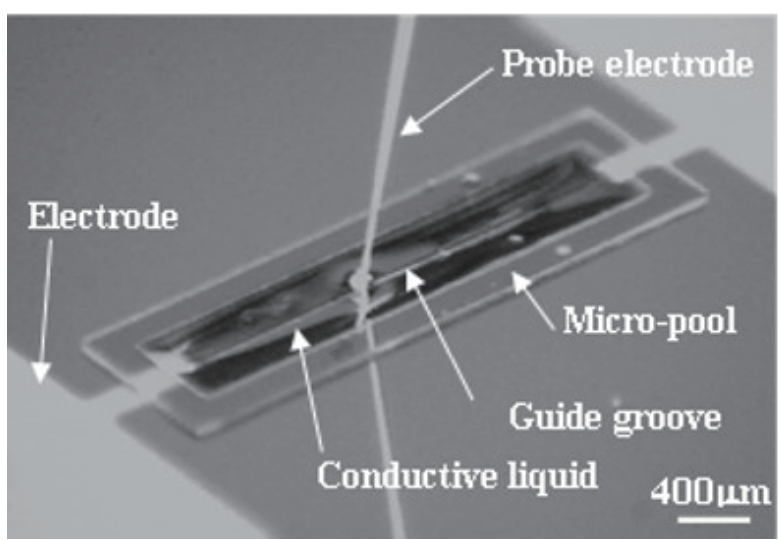

(b)

Fig. 3. Fabricated devices. (a) Fabricated Si strip with micropools and electrodes. (b) Magnification of interior of the microlinear potentiometer. 


\section{Characterization}

\subsection{Fundamental property}

The proposed connection can overcome the drawbacks of conventional electrical connections, such as the physical restriction caused by a suspension spring for microactuators, and enables the connection distance to be extended without increasing restriction. For example, electrostatic comb drive actuators made of $\mathrm{Si}$ are supported by $\mathrm{Si}$ suspension springs to supply driving signals. Si suspension springs were prepared for comparison with the developed sliding connection using a conductive liquid. Table 1 presents individual specifications of the sliding contact with a micropool of conductive liquid and the Si suspension spring. Figure 4(a) shows the images of the setup used to evaluate the sliding contact and the Si suspension spring. The physical restriction force was measured with a load cell (LVS-10GA, KYAWA). The probe was moved through the electroconductive liquid at $5 \mu \mathrm{m} / \mathrm{s}$. The physical restriction force of the tension of the Si spring increased proportionally to displacement, whereas the fluid resistance against the probe electrode in the micropool was approximately $3 \mathrm{mN}$, as shown in Fig. 4(b).

Table 1

Specifications of the sliding contact with micropool with conductive liquid and the Si suspension spring.

\begin{tabular}{lcccc}
\hline & Width $(\mu \mathrm{m})$ & Length $(\mu \mathrm{m})$ & Depth $(\mu \mathrm{m})$ & Diameter of probe $(\mu \mathrm{m})$ \\
\hline $\begin{array}{l}\text { Sliding connection with } \\
\text { micropool of conductive }\end{array}$ & 1000 & 2000 & 150 & 20 \\
liquid & Width $w(\mu \mathrm{m})$ & Length $l(\mu \mathrm{m})$ & Thickness $t(\mu \mathrm{m})$ & Number of springs \\
\hline & 250 & 1900 & 55 & 13 \\
\hline Si suspension spring & 250 &
\end{tabular}

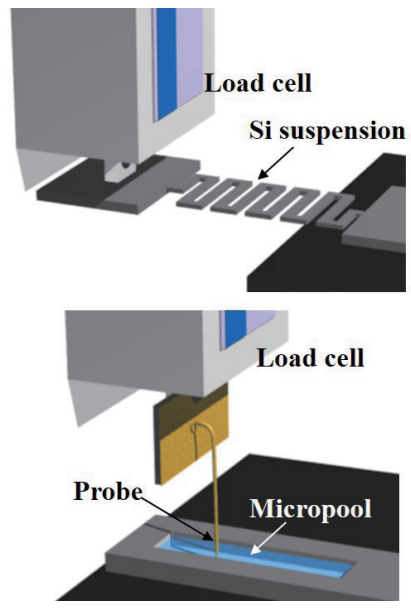

(a)

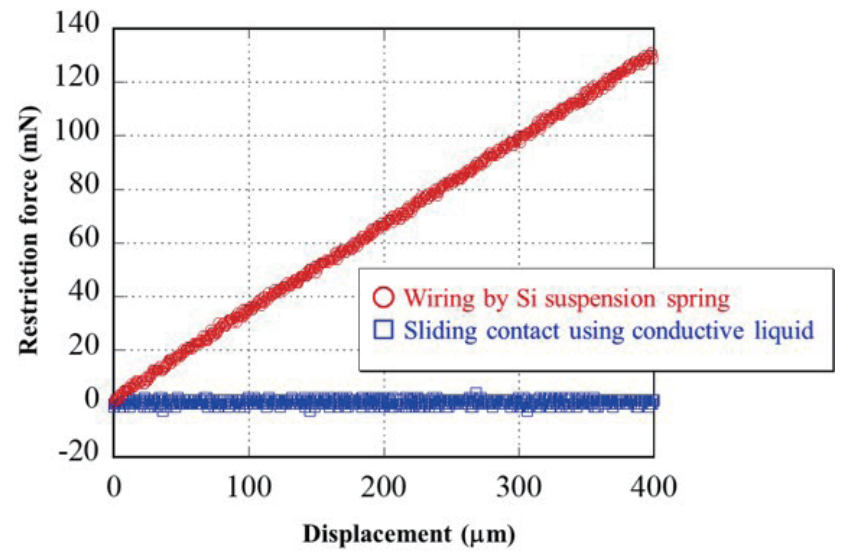

(b)

Fig. 4. (Color online) Restriction force of the sliding contact in comparison with Si suspension springs. (a) Images of evaluation setup and (b) evaluated results of restriction force. 
The proposed micropotentiometer was characterized as a displacement sensor. Figure 5 shows the results of the electrical resistance change against displacement. A voltage of $1 \mathrm{~V}$ was applied across the two terminal electrodes at the edges of the micropool by taking into account a potential window of the ionic liquid. The probe was moved using an external positioning control system. In the experiments, we obtained repeatable linear characteristics without an obvious hysteresis, as shown in Fig. 5. The initial resistance was governed by the gap between the probe electrode and the terminal electrode. A detailed analysis of the linearity revealed that the maximum linearity error was $1.7 \%$. The error includes the influence of the probe motion driven by an external positioning system. The electrical resistance depends on the relative distance between the probe and the terminal electrodes. The resistance change affects the linearity of the resolution because it depends on the depth of the probe. The developed potentiometer could detect displacements of $2 \mu \mathrm{m}$ with good linearity. In addition, the potentiometer detected reproducible characteristics in cases where the applied voltage between the two terminal electrodes was within the potential window of the ionic liquid.

The physical restriction force against the displacement of the wiper was estimated in Fig. 4. A commercialized potentiometer $\left(8 \times 30 \times 7 \mathrm{~mm}^{3}\right.$, WAPAOB503C15, Tyco Electronics $)$ was, herein, compared with our potentiometer. The motion velocity of the probe was set at 5 $\mu \mathrm{m} / \mathrm{s}$. The measured restriction force was approximately $3 \mathrm{mN}$, whereas the commercialized potentiometer required more than $100 \mathrm{mN}$. The result shows that the proposed potentiometer with an electroconductive liquid could markedly reduce the physical restriction.

\subsection{Reliability}

Potentiometers are often applied to actuators to detect their motion. Wipers of potentiometers move together with the actuators. Potentiometers are required to guarantee a stable performance in any position or attitude. The proposed micropotentiometer employs

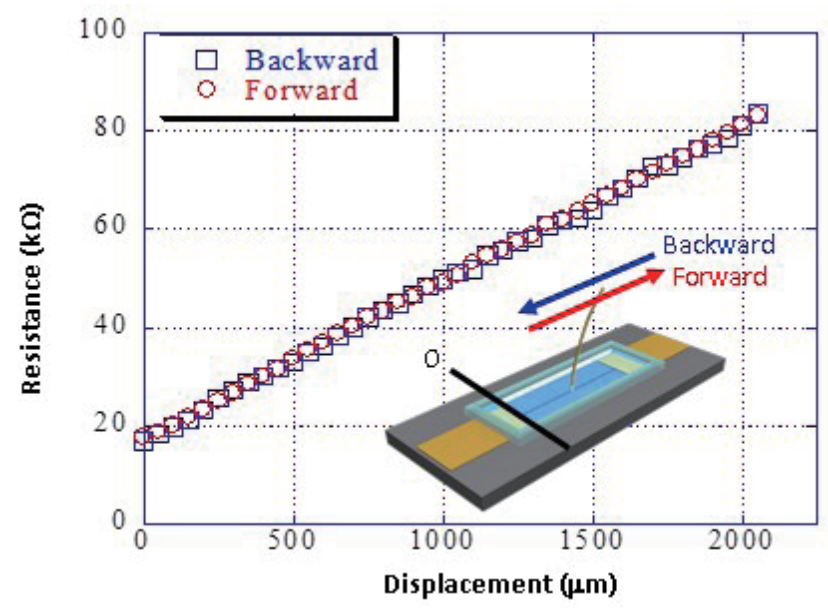

Fig. 5. (Color online) Characterization of developed microlinear potentiometer. Both forward and backward motions were estimated. 
a conductive liquid as a resistor. In this study, an ionic liquid, 1-ethyl-3-methylimidazolium trifluoroacetate, was employed as the conductive liquid and introduced into the micropool fabricated in a Si chip. 1-ethyl-3-methylimidazolium trifluoroacetate was reported in the early $90 \mathrm{~s}$ as the attractive material used to overcome drawbacks of a conventional unstable ionic liquid. It improved the durability against water and the low causticity to the metal. This study started with 1-ethyl-3-methylimidazolium trifluoroacetate as the representative ionic liquid. As a matter of course, there is a room to optimize the ionic liquid depending on the purpose. For example, it becomes important to design the hydrophilicity of the ionic liquid to retain the ionic liquid in the device in the long term. At the same time, the wettability control of the structure is also interesting in terms of introducing and retaining the conductive liquid in the micropool for the high durability. In general, the Si surface is rather hydrophilic and its contact angle increases with its surface roughness through plasma etching. It is possible to control the wettability of the device in accordance with the purpose. The ionic liquid was evaluated by cyclic voltammetry. As a result, a stable potential region was estimated between -2 and $+3 \mathrm{~V}$ within the potential window. Figure 6 shows the measured dividing voltages when the applied potential was increased from 2 to $4 \mathrm{~V}$ and $10 \mathrm{~V}$. The measured signal at $2 \mathrm{~V}$ was stable, whereas signals at 4 and $10 \mathrm{~V}$ were unstable owing to the available range restricted by the potential window.

It is also important to verify the stability in various attitudes. The long-term stability is also important. The stability of the linear micropotentiometer was verified by inclination and longterm tests. We verified its effect on detection using a liquid for the electrical connection (Fig. 7). The fabricated potentiometer was set on an inclined stage $\left(45,90^{\circ}\right)$ and moved using an external positioning control system. Characteristics under a flat condition $\left(0^{\circ}\right)$ were also estimated as a control. There were no obvious changes in the characteristics as the inclination angle was varied ( 0 , $45,90^{\circ}$ ). The conductive liquid in the micropool did not spill because of a surface tension.

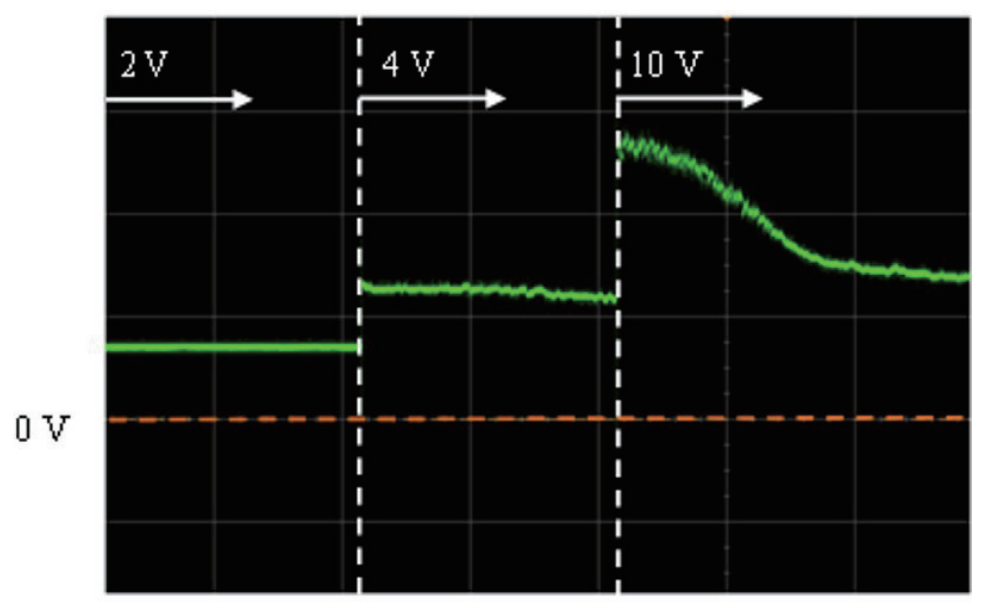

Fig. 6. (Color online) Stability evaluation depending on the potential window of ionic liquid ( $X: 10 \mathrm{~s} / \mathrm{div}, Y: 2$ V/div). The dividing voltage was measured when the applied voltage was increased beyond the potential window. 


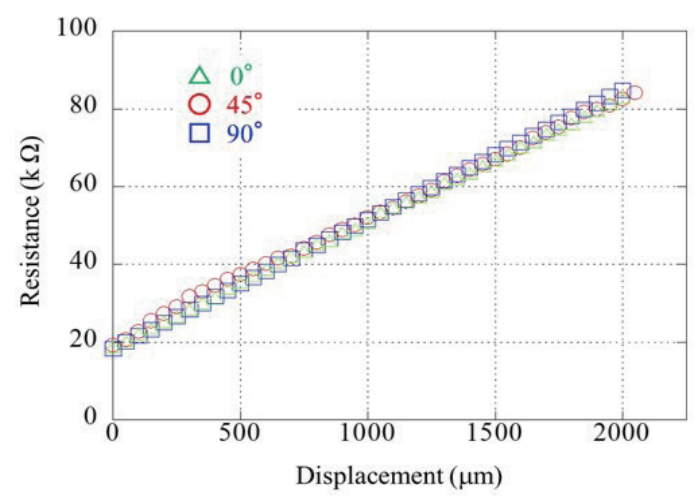

Fig. 7. (Color online) Evaluation results of tilting effect. Inclinations of 0,45 and $90^{\circ}$ were used.

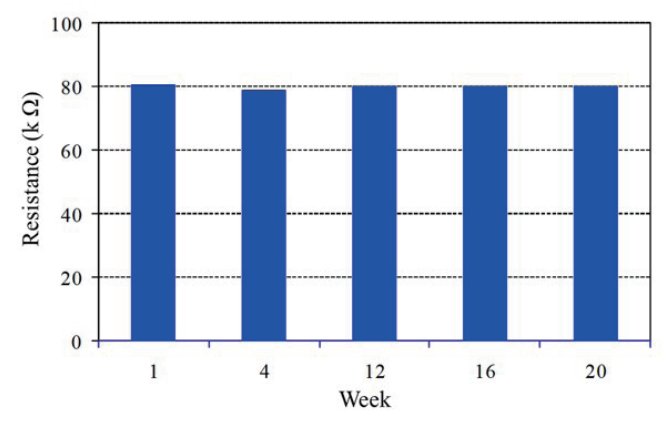

Fig. 8. (Color online) Evaluation of long-term stability of the micropotentiometer.

Next, we performed long-term tests to confirm the long-term stability of the fabricated potentiometer (Fig. 8). The resistance of the potentiometer was measured for 20 weeks under fixed environmental conditions (humidity: $18 \%$, temperature: $20{ }^{\circ} \mathrm{C}$ ). The characteristics showed a maximum change of $0.8 \%$ (1 week: $80.86 \mathrm{k} \Omega, 14$ weeks: $80.21 \mathrm{k} \Omega$ ). These results indicate that the potentiometer exhibits a long-term stability.

\section{Conclusions}

A sliding electrical contact with an electroconductive liquid was presented. The proposed sliding contact was a combination of an electroconductive liquid in a micropool as one electrode and a probe electrode dipped into the micropool as the movable electrode. A nonvolatile, ionic liquid, 1-ethyl-3-methylimidazolium trifluoroacetate, was employed as the electroconductive liquid. The probe electrode was fabricated by a combination of wire bonding and laser machining. The proposed sliding contact reduced physical restriction, such as sliding friction at the contact point, which enabled the connection distance to be extended without increasing restriction. As a typical application of the proposed sliding contact, a micropotentiometer was demonstrated. In this micropotentiometer, the ionic liquid in the micropool and the dipped probe function served as the resistor and wiper, respectively. The proposed micropotentiometer significantly reduced the physical restrictions at the contact point of variable resistances. A $500 \times 2500 \times 70 \mu \mathrm{m}^{3}$ micropool and a $20-\mu \mathrm{m}$-diameter $\mathrm{Au}$ wire were prepared for the micropotentiometer. The micropotentiometer could detect displacements of $2 \mu \mathrm{m}$ with good linearity. The measured restriction force was only about $3 \mathrm{mN}$ when the probe was moved in the ionic liquid at $5 \mu \mathrm{m} / \mathrm{s}$, whereas the value for a commercial potentiometer was more than 100 $\mathrm{mN}$. Moreover, the inclination and long-term tests guaranteed the reliability of the proposed micropotentiometer. 


\title{
Acknowledgments
}

This work was partially supported by the Adaptable and Seamless Technology Transfer Program through Target-driven R\&D (A-STEP), JST. The author gratefully acknowledges the helpful assistance of Mr. M. Ito in carrying out this study. The author also wish to thank Mr. T. Kuwamura and Mr. K. Ishi for their helpful assistance with this study.

\section{References}

1 S. Suzuki, S. Tuchitani, K. Sato, S. Ueno, Y. Yokota, M. Sato, and M.Esashi: Sens. Actuators, A $21-23$ (1990) 316.

2 T. Hirano, T. Furuhata, K. J. Gabriel, and H. Fujita: J. Microelectromech. Syst. 1 (1992) 52.

3 R. Legtenberg, A. W. Groeneveld, and M. Elwenspoek: J. Micromech. Microeng. 6 (1996) 320.

4 X. Wang, X. Wang, H. Lu, F. Qian, and Y. Bu: Opt. Laser Technol. 33 (2001) 219.

5 S. J. Lee, Y. Melikhov, D. C. Jiles, C. M. Park, and H. Hauser: J. Appl. Phys. 99 (2006) 08 B301.

6 W. Gao, S. Dejima, and S. Kiyono: Sens. Actuators, A 17 (2005) 95.

7 M. Ito, T. Maeda, K. Shimizu, and S. Konishi: Proc. Nat. Conf. IEEJ (2010) 175.

8 T. Liu, G. Sun, J. J. Kim, C.-K. K. Yang, and C.-J. Kim: Proc. IEEE Int. Conf. MEMS (2013) 86-89.

9 K. Noda, K. Matsumoto, and I. Shimoyama: Sens. Actuators, A 215 (2014) 123.

10 M. Ito, Y. Kuwamura, and S. Konishi: Proc. IEEE Int. Conf. MEMS (2010) 228-291.

11 K. Shimizu, M. Nakanishi, M. Makikawa, S. Asajima, and S. Konishi: IEEJ Trans. Sens. Micro-machines 129 (2009) 387.

12 W. Tonomura, K. Shimizu, and S. Konishi: Proc. IEEE Int. Conf. MEMS (2009) 741-744.

\begin{abstract}
About the Author
Satoshi Konishi received his B.S. degree in electronics engineering in 1991 and his M.S. and Ph.D. degrees in electrical engineering in 1993 and 1996, respectively, all from the University of Tokyo, Tokyo, Japan. He has been a professor at Ritsumeikan University, Shiga, Japan, since 2006, where he joined the faculty in 1996. He is an editorial board member of Sensors and Actuators A, Journal of Micromechanics and Microengineering, and Microsystems \& Nanoengineering, and an editor of the IEEE Journal of Microelectromechanical Systems. He is also an international steering committee member of Transducers and IEEE International Conference of MEMS. He served as a general co-chair of International Conference of MEMS 2007 in Kobe. His study is devoted to micro-electromechanical systems (MEMS), covering broad ranges from fundamental to applied fields. His current research focuses on biomedical applications based on microrobots and cyborgs. (konishi@se.ritsumei.ac.jp)
\end{abstract}

